\title{
A mettre sur votre "reading list» si ce n'est pas encore fait
}

\section{Jean Martin}

Dr méd., membre de la rédaction

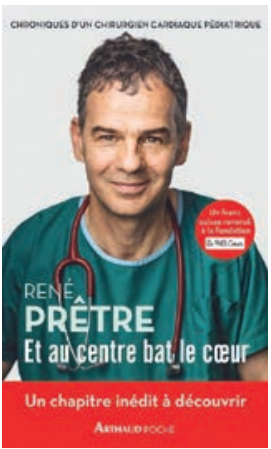

René Prêtre

Et au centre bat le cœur

(Réédition avec un chapitre inédit)

Paris: Arthaud Poche; 2018

358 pages. $31.90 \mathrm{CHF}$.

ISBN 978-2081420014

Ce bestseller du "Suisse de l'année 2009" a paru à l'automne 2016. J'avais entendu parler de son succès mais ne l'avais pas lu. Trop d'autres lectures? Réticence vis-àvis du récit, peut-être "avantageux", des hauts faits d'une super-star? Quoi qu'il en soit, ce n'était pas bien, ce livre est remarquable.

L'histoire de René Prêtre (né en 1957) est connue. Enfant d'une famille paysanne du Jura, footballeur passionné (des primes reçues l'ont quelque peu aidé durant ses études!), il est devenu un grand de la chirurgie cardiaque. Etudes et formation initiale à Genève, puis New York aux urgences du Bellevue Hospital - dont on dit «Ce qui n’a pas été vu au Bellevue n'existe peut-être pas»! Des stages dans plusieurs autres pays, puis dès 1997 à l'Hôpital universitaire de Zurich, où il devient chef de la chirurgie cardiaque pédiatrique. Patron de la chirurgie cardio-vasculaire au CHUV de Lausanne depuis 2012, puis aussi à Genève.

Récit en 19 chapitres. L'enfance de Boncourt puis formation et ascension dans la carrière professionnelle et académique. Avec des missions dans des pays en développement (ceci alors que Prêtre dit sa réticence initiale à "exporter" une médecine hautement technologique, exigeant de gros moyens, dans des endroits où les besoins généraux sont massifs, en termes de santé publique et d'éducation en particulier - judicieuse réflexion).

L'auteur a au cours des années dicté son vécu des histoires rencontrées, souvent compliquées, parfois déchirantes; les côtés satisfaisants voire enthousiasmants, mais aussi les soucis, la charge de travail (devenir chirurgien cardiaque, c'est travailler dix heures par jour durant dix ans, disait un de ses maîtres). Fierté de l'opération réussie dans les cas les plus trapus. Ce qui marque lecteurs et journalistes (j'ai passé un peu de temps à «googler»), c'est comment il raconte quelques

échecs lourds, traumatisants - ainsi la complication, aux suites handicapantes, de Robin, garçon dont il avait convaincu la mère de pratiquer une opération qui n'était pas vitale sur le moment (la date de cet échec restant, chaque année, un funeste anniversaire).

Ces accidents de parcours, racontés avec beaucoup de transparence, sont en contrepoint d'une carrière à succès, qui à l'évidence sort de l'ordinaire. Il évoque les dimensions éthiques de son activité, discutées en équipe. Décrit aussi en détail des dialogues difficiles avec des parents désemparés devant les enjeux.

La manière dont Prêtre parle de ses équipes donne envie d'en faire partie. Comme d'autres, il a probablement aussi ses colères ou ses bougonnements mais on retire l'impression que le plus souvent, y compris quand cela "coince», voire qu'il y a urgence extrême, une collaboration entraînée, affinée au cours des années, fait que les gens sont heureux de tirer à la même corde (je ne le connais pas personnellement, peut-être suis-je trop ébloui?).

J'ai été frappé par la qualité de la rédaction. C'est fort bien écrit... une vraie plume. Pas de moments où on s'ennuie - et pas de culte de la personnalité.

Ce livre est un "page turner» (on ne résiste guère à l'envie de poursuivre la lecture). En exergue de chaque chapitre, une phrase tirée d'une oeuvre littéraire, d'un opéra ou d'ailleurs! Dans une vie consacrée massivement au métier, on imagine que René Prêtre trouve le temps de lire (autre chose que de la science) et d'écouter de la musique. Quelques lignes de citation pour finir: "Ainsi, les heures à la mine m'apprirent à ériger ces deux piliers essentiels en chirurgie: la technique et la stratégie. J'allais en découvrir plus tard un troisième, la dimension artistique, la maitrise de l'espace, des formes (...) Cette chorégraphie fluide et cette détermination me firent comprendre que la fabrication d'un chirurgien passait par un modelage long et astreignant de ses doigts et de son esprit.» Et aussi: «Les opérations néonatales et leurs grandeurs réduites renforçaient encore cette impression d'entrer dans le cœur de la vie. L'intérieur de ces thorax n'apparaissait plus comme une simple salle des machines mais irradiait d'un magnétisme particulier, touchant au fantastique.» 